\title{
Immunohistochemical and molecular detection of the expression of FGF23 in phosphaturic mesenchymal tumors including the non-phosphaturic variant
}

Eisuke Shiba', Atsuji Matsuyama ${ }^{1 *}$, Ryo Shibuya ${ }^{1}$, Kei Yabuki ${ }^{1}$, Hiroshi Harada ${ }^{1}$, Mitsuhiro Nakamoto²,

Takahiko Kasai ${ }^{3}$ and Masanori Hisaoka ${ }^{1}$

\begin{abstract}
Background: Phosphaturic mesenchymal tumors (PMTs) are rare neoplasms that are often associated with tumor-induced osteomalacia (TIO) due to excessive serum levels of fibroblast growth factor 23 (FGF23). PMTs share overlapping histologic features with other types of tumors; thus, accurate pathological diagnosis may be challenging. We performed an immunohistochemical examination of FGF23 expression in PMTs and other types of tumors, together with pertinent molecular analyses.

Methods: Seven PMTs (5 with $\mathrm{TIO}$ and 2 without $\mathrm{TIO}$ ) and 46 other types of bone and soft tissue tumors were retrieved, and immunohistochemistry was performed using a commercially available anti-FGF23 antibody. In addition, FGF23 mRNA expression was detected by reverse transcription-polymerase chain reaction (RT-PCR), using RNA extracted from formalin-fixed, paraffin-embedded tissues.

Results: Immunohistochemical analysis of FGF23 expression showed distinct, punctate staining in the cytoplasm in 5 PMTs with TIO, whereas FGF23 expression was negative in the 2 PMTs without TIO and the other 46 tumors. FGF23 mRNA expression was detected in all 4 PMTs examined, as well as in 1 chondromyxoid fibroma and 1 myxoid liposarcoma. The real-time RT-PCR data showed that the relative expression levels of the FGF23 mRNA tended to be higher in PMTs with $\mathrm{TIO}$ than in PMTs without TIO, or in the chondromyxoid fibroma specimen.

Conclusions: Our data suggested that the feasibility of immunohistochemical detection of FGF23 may depend on the level of secreted FGF23 from tumor cells. Thus, immunohistochemistry for FGF23 is an useful diagnostic adjunct for PMT, although its utility appears to be limited in cases without TIO.
\end{abstract}

Keywords: Phosphaturic mesenchymal tumor, Tumor-induced osteomalacia, Non-phosphaturic variant, Fibroblast growth factor 23, Immunohistochemistry, Polymerase chain reaction

\section{Background}

Tumor-induced osteomalacia (TIO) was previously described as involving various types of bone and soft tissue tumors such as hemangiopericytoma (an obsolete term, which is now referred to as solitary fibrous tumor), chondroblastoma, and osteosarcoma [1-5]. Recent

\footnotetext{
* Correspondence: amatsu@med.uoeh-u.ac.jp

${ }^{1}$ Department of Pathology and Oncology, School of Medicine, University of Occupational and Environmental Health, 1-1 Iseigaoka, Yahatanishi-ku, Kitakyushu 807-8555, Japan

Full list of author information is available at the end of the article
}

findings have revealed that the majority of such cases encompasses a single entity with distinctive histological features, and this specific group of lesions has been designated as phosphaturic mesenchymal tumor (PMT) [1-3]. The formation of TIO in PMTs is thought to result from an excessive serum level of tumor-derived fibroblast growth factor 23 (FGF23) [3-5], which is an osteocyte-derived phosphaturic hormone that inhibits renal phosphate reabsorption and the production of $1,25(\mathrm{OH})_{2}$ vitamin $\mathrm{D}$ by reducing the activity of renal $1 \alpha$-hydroxylase [6-8]. However, rare PMTs likely express 
Table 1 Immunohistochemical results of FGF23

\begin{tabular}{ll}
\hline Diagnosis & Positive \\
\hline Phosphaturic mesenchymal tumor & $5 / 7$ \\
Chondromyxoid fibroma & $0 / 6$ \\
Chondroblastoma & $0 / 4$ \\
Chondrosarcoma & $0 / 4$ \\
Extraskeletal mesenchymal chondrosarcoma & $0 / 1$ \\
Osteosarcoma & $0 / 5$ \\
Synovial sarcoma & $0 / 3$ \\
Angiosarcoma & $0 / 2$ \\
Clear cell sarcoma & $0 / 2$ \\
Myxoid liposarcoma & $0 / 1$ \\
Solitary fibrous tumor & $0 / 4$ \\
Giant cell tumor of bone & $0 / 4$ \\
Giant cell tumor of tendon sheath & $0 / 6$ \\
Aneurysmal bone cyst & $0 / 4$ \\
\hline
\end{tabular}

other phosphaturic hormones (e.g., frizzled-related protein 4 and matrix extracellular phosphoglycoprotein) [9-11]. The minor fraction of PMTs that lack clinical evidence of $\mathrm{TIO}$ is referred to as the non-phosphaturic variant [3].

In 2009, Bahrami et al. [12] reported the diagnostic utility of detecting FGF23 transcripts in PMTs by the reverse transcription-polymerase chain reaction (RT-PCR) using formalin-fixed, paraffin-embedded (FFPE) tumor tissues. Although RT-PCR testing is highly sensitive, FGF23 mRNA is considered to be structurally normal and could also be detected in non-PMT tumors, including aneurysmal bone cysts and chondromyxoid fibromas [12-14]. A more reliable diagnostic adjunct for routine pathology testing is required.

Here, we performed immunohistochemical staining for FGF23 expression in PMTs, with or without TIO, and other types of bone and soft tissue tumors using a commercially available anti-FGF23 antibody together with real-time RT-PCR, This approach allowed us to address differences in FGF23 expression between PMTs, with and without TIO.

Table 2 Results of the cases evaluated by a RT-PCR analysis

\begin{tabular}{|c|c|c|c|c|c|c|}
\hline No & $\begin{array}{l}\text { Age } \\
(y) / \\
\text { sex }\end{array}$ & Site & Diagnosis & $\mathrm{TIO}$ & $\mathrm{IHC}$ & $\begin{array}{l}\text { RT-PCR } \\
\text { FGF23a/b/c }\end{array}$ \\
\hline 1 & $35 / F$ & Popliteal region & Phosphaturic mesenchymal tumor & + & + & $+/+/+$ \\
\hline 2 & $59 / F$ & Groin & Phosphaturic mesenchymal tumor & + & + & $+/+/+$ \\
\hline 3 & $59 / F$ & Nasal cavity & Phosphaturic mesenchymal tumor & + & + & NA \\
\hline 4 & $61 / \mathrm{M}$ & Lower leg & Phosphaturic mesenchymal tumor & + & + & NA \\
\hline 5 & $46 / F$ & Nasal cavity & Phosphaturic mesenchymal tumor & + & + & NA \\
\hline 6 & $38 / F$ & Foot & Phosphaturic mesenchymal tumor & - & - & $+/+/+$ \\
\hline 7 & $43 / M$ & Radius & Phosphaturic mesenchymal tumor & - & - & $+/+/+$ \\
\hline 8 & $57 / \mathrm{M}$ & Distal phalanx & Chondromyxoid fibroma & & - & NA \\
\hline 9 & 30/M & Fibula & Chondromyxoid fibroma & & - & $+/-/+$ \\
\hline 10 & 23/M & Patella & Chondroblastoma & & - & $-/-/-$ \\
\hline 11 & 15/M & Humerus & Chondroblastoma & & - & $-/-/-$ \\
\hline 12 & $26 / M$ & Calcaneus & Chondroblastoma & & - & $-/-/-$ \\
\hline 13 & $57 / M$ & Tibia & Chondroblastoma & & - & $-/-/-$ \\
\hline 14 & $74 / F$ & Middle finger & Giant cell tumor of tendon sheath & & - & $-/-/-$ \\
\hline 15 & $66 / M$ & Index finger & Giant cell tumor of tendon sheath & & - & $-/-/-$ \\
\hline 16 & $51 / F$ & Wrist & Giant cell tumor of tendon sheath & & - & $-/-/-$ \\
\hline 17 & $62 / F$ & Femur & Giant cell tumor of bone & & - & $-/-/-$ \\
\hline 18 & 39/M & Femur & Giant cell tumor of bone & & - & $-/-/-$ \\
\hline 19 & 70/F & Femur & Aneurysmal bone cyst & & - & $-/-/-$ \\
\hline 20 & $74 / F$ & Femur & Aneurysmal bone cyst & & - & $-/-/-$ \\
\hline 21 & $71 / F$ & Vertebra & Aneurysmal bone cyst & & - & $-/-/-$ \\
\hline 22 & 20/M & Thigh & Extraskeletal mesenchymal chondrosarcoma & & - & $-/-/-$ \\
\hline 23 & $65 / F$ & Lower leg & Myxoid liposarcoma & & - & $-/+/-$ \\
\hline
\end{tabular}

$\overparen{I H C}$ immunohistochemistry, NA not available, RT-PCR reverse transcription-polymerase chain reaction, $T I O$ tumor-induced osteomalacia 


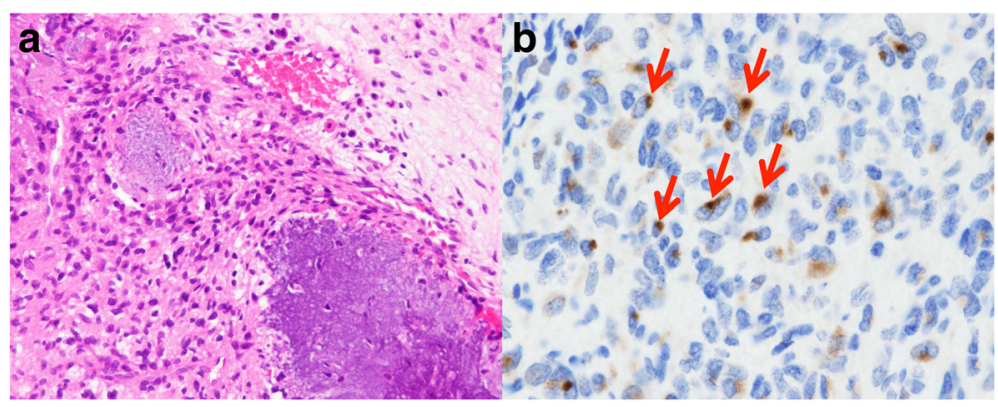

Fig. 1 PMT with TIO. a: The tumor was characterized by the proliferation of bland spindle or oval cells, with grungy calcification. b: By immunohistochemical staining, FG322-3 expression showed a distinct, dot-like staining pattern (arrows)

\section{Methods}

Archived specimens from 7 PMTs (5 with TIO and 2 without TIO) and 46 other bone and soft tissue tumors (6 chondromyxoid fibromas, 4 chondroblastomas, 4 chondrosarcomas, 1 extraskeletal mesenchymal chondrosarcoma, 5 osteosarcomas, 3 synovial sarcomas, 2 angiosarcomas, 2 clear cell sarcomas, 1 myxoid liposarcoma, 4 solitary fibrous tumors, 4 giant cell tumors of the bone, 6 giant cell tumors of the tendon sheath, and 4 aneurysmal bone cysts) were obtained from our institution. PMT diagnosis was made based on clinical information and morphological findings, including dirty or smudgy calcification, as determined by two pathologists (M.H. \& A.M.). For immunohistochemical examinations, histological sections of FFPE tumor specimens were incubated with an anti-FGF23 monoclonal antibody (FG322-3, 1:500 dilution; Adipogen, San Diego, CA, USA) at room temperature for $24 \mathrm{~h}$ after epitope retrieval in ethylenediaminetetraacetic acid buffer $(\mathrm{pH}$ 8.0) using a pressure cooker, followed by treatment with $3 \%$ hydrogen peroxide for $10 \mathrm{~min}$. Immunostaining was accomplished by incubation with a labeled polymeric secondary antibody (Histofine Simple stain MAX PO, Nichirei, Tokyo, Japan). Diaminobenzidine solution was used for visualization, followed by nuclear counterstaining with hematoxylin. As described by Nelson et al. [15] and Houang et al. [16], distinct "dot-like" cytoplasmic staining of FGF23 was considered to represent a positive result, whereas diffuse cytoplasmic and nuclear staining were interpreted as non-specific findings.

For molecular detection of the FGF23 gene transcript, total RNA was extracted from FFPE tissues using the TRIzol reagent (Invitrogen, Carlsbad, CA, USA) and reverse transcribed into cDNA. RT-PCR-based analysis of the FGF23 transcripts was performed using 3 different sets of primers designed by Bahrami et al. [12]. The transcripts of reference genes (phosphoglycerate kinase and porphobilinogen deaminase) were amplified along with FGF23 as quality controls. Quantitative analysis of FGF23 mRNA expression was performed by real-time RT-PCR analysis using a TaqMan Gene Expression Assay (Applied Biosystems, Foster City, CA, USA), according to the manufacturer's instructions. Briefly, 20- $\mu \mathrm{l}$ PCR reaction mixtures containing $1 \times$ TaqMan Gene Expression Master Mix, 1× TaqMan Gene Expression Assay, and the reverse transcription products were incubated at $95{ }^{\circ} \mathrm{C}$ for $10 \mathrm{~min}$, followed by 40 cycles at $95{ }^{\circ} \mathrm{C}$ for $15 \mathrm{~s}$ and at $60{ }^{\circ} \mathrm{C}$ for $1 \mathrm{~min}$. Standard curves were generated to quantitate the data. FGF23 mRNA expression levels were normalized to that of the glyceraldehyde 3-phosphate dehydrogenase $(G A P D H)$ gene, as an endogenous control.

The study design was approved by an ethics review board of the University of Occupational and Environmental Health (H25-169).

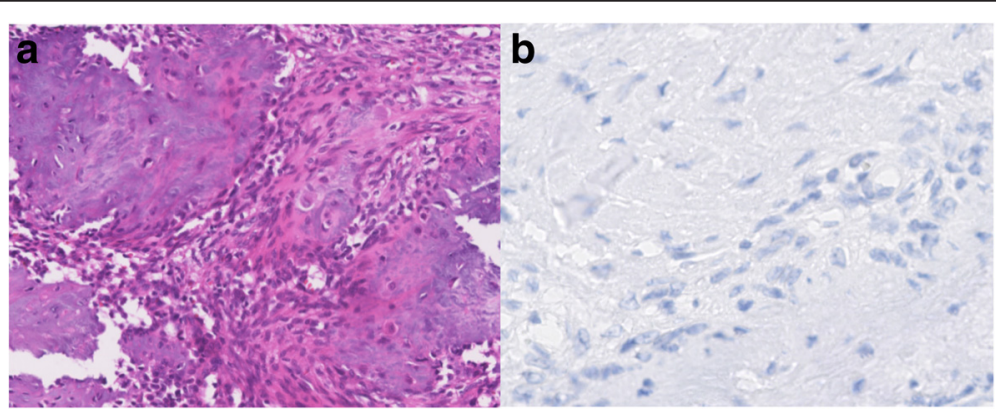

Fig. 2 PMT without TIO. a: The tumor was characterized by the proliferation of bland spindle cells, with grungy calcification. b: Immunohistochemical staining showed that the tumor cells were negative for FG322-3 


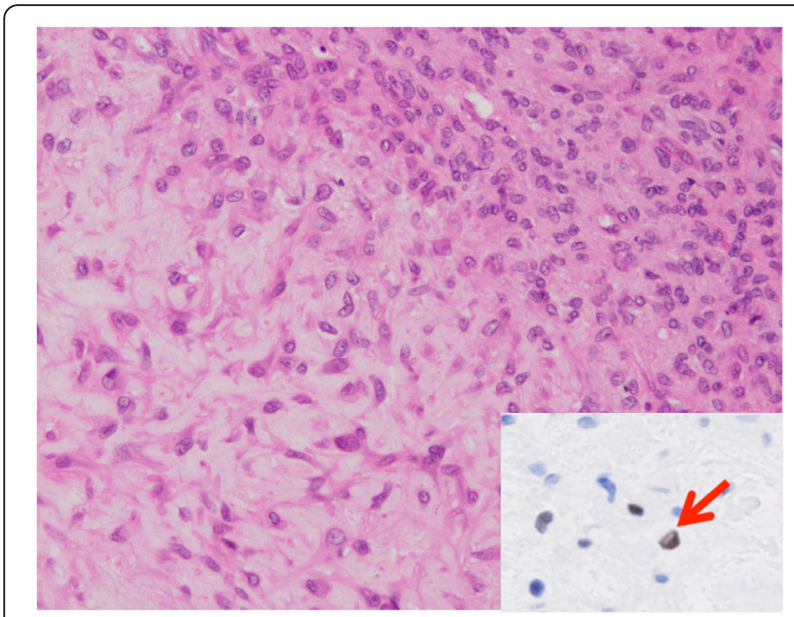

Fig. 3 Chondromyxoid fibroma. Inset: Weak nuclear staining of FG322-3 was noted in a small proportion of the tumor cells (arrow)

\section{Results and discussion}

The results of our immunohistochemistry analysis of FGF23 expression in the tumor specimens examined are summarized in Table 1. Five of 7 (71.4 \%) PMTs were positive for FG322-3, and showed a distinct, punctate intracytoplasmic and perinuclear staining pattern in 5$20 \%$ of the tumor cells (Fig. 1). Most positive cells were oval or spindle-shaped and tended to reside in densely populated cellular areas, rather than hypocellular calcified areas. All 5 of the FG322-3-positive PMTs had TIO. In contrast, no FG322-3-positive cells were observed among the 2 PMTs without TIO (Fig. 2) or the 46 non-PMT tumors, although non-specific nuclear and/or diffuse cytoplasmic staining was noted in 9 cases (Figs. 3 and 4).

Results of the cases evaluated by a RT-PCR analysis are summarized in Table 2. During RT-PCR analysis, FGF23 gene transcripts were identified in all 4 PMTs examined, 2 of which were associated with TIO (Fig. 5). Only 2 of the 3 FGF23 transcripts examined were detected in a chondromyxoid fibroma, and 1 of the 3 studied transcripts was identified in a myxoid liposarcoma
(Fig. 5). No FGF23 transcript was amplified in the other tumors examined. Analysis of our real-time RT-PCR data showed that the relative expression levels of the FGF23 gene transcripts tended to be higher in PMTs with TIO than those without TIO, or in chondromyxoid fibroma samples (Fig. 6). The FGF23 gene transcript was not amplified in the other tumor specimens examined, including the myxoid liposarcoma specimen (Fig. 6).

PMT is a rare neoplasm of soft tissues and bones that arises predominantly in middle-aged adults and generally has a benign clinical course [3-5]. The characteristic morphological features of PMTs include bland spindle cell cytomorphology, a low mitotic activity, grungy or flocculent calcification, and a myxoid or myxochondroid stroma [3-5]. Osteoclast-like giant cells, microcystic changes, an osteoid matrix, hemangiopericytomatous blood vessels, and a mature adipose-tissue component may be present in some cases [3-5]. However, some PMTs may contain areas of high-grade sarcoma resembling undifferentiated pleomorphic sarcoma or fibrosarcoma and often behave in an aggressive manner. In addition, histologically benign PMTs may also metastasize [17-20]. An accurate diagnosis of PMT is often difficult due to histological heterogeneity, and differential diagnoses include many other types of tumors with some similar morphologic features, such as chondromyxoid fibroma, chondroblastoma, aneurysmal bone cyst, and osteosarcoma.

In this study, we found that the immunohistochemical expression of FGF23 was highly specific for PMTs with TIO (100\%). The sensitivity was $71.4 \%$ in our overall series of PMTs tested, and $100 \%$ in the phosphaturic variant. Although the number of examined tumor samples was small, our data suggest that FGF23 is a useful immunohistochemical marker for PMT with TIO. It is important to note that only the punctate staining pattern was considered to be positive, similar to previous studies using other anti-FGF23 antibody clones [15, 16]. Diffuse cytoplasmic and nuclear staining are occasionally encountered in non-PMT tumors or normal tissues at inappropriately high antibody concentrations, whereas

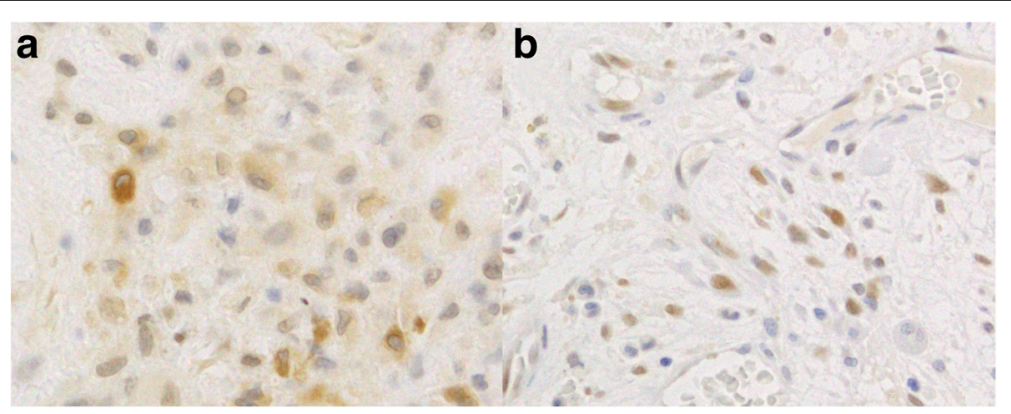

Fig. 4 Non-specific FG322-3 immunostaining observed using a high antibody concentration. a: Diffuse cytoplasmic staining in a chondromyxoid fibroma. b: Nuclear staining in an aneurysmal bone cyst 


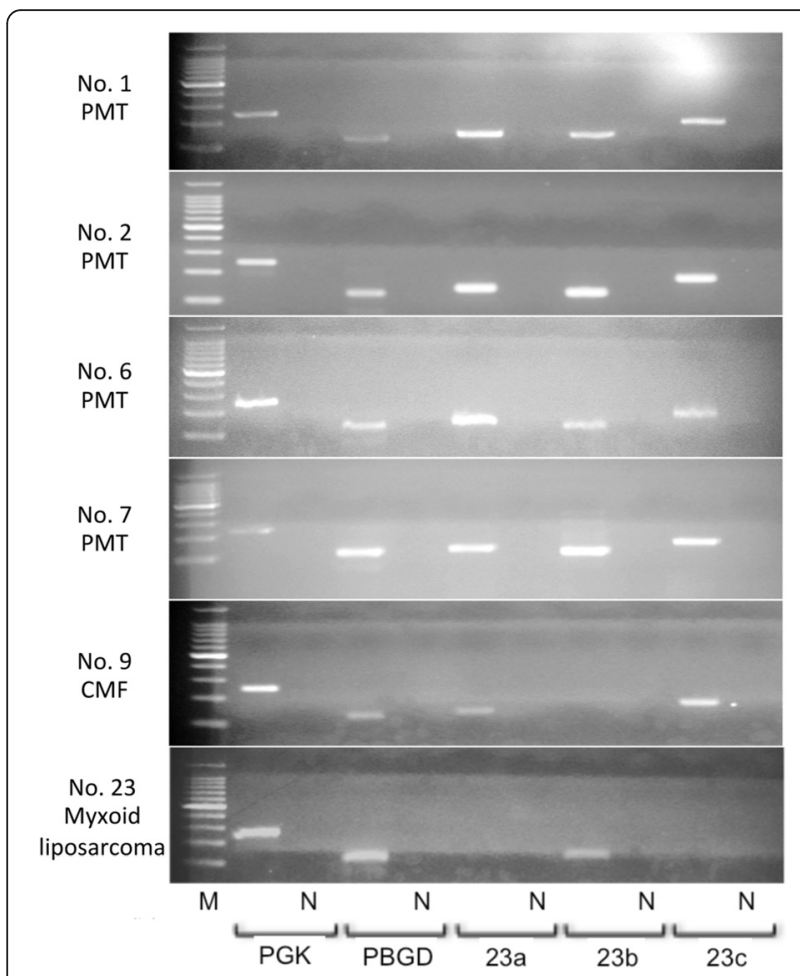

Fig. 5 RT-PCR analysis of FGF23 transcripts. All 3 FGF23 transcripts (23a, 140 bp; 23b, 125 bp; 23c, 175 bp) were amplified in PMTs (with TIO: case Nos. 1, 2, without TIO: case Nos. 6, 7), whereas only 2 or 1 of the 3 transcripts were detected in the chondromyxoid fibroma sample (case No. 9) or the myxoid liposarcoma sample (case No. 23), respectively. M, 100-bp DNA ladder; N, negative control; PGK, phosphoglycerokinase; PBGD, porphobilinogen deaminase; CMF, chondromyxoid fibroma such staining patterns are inconspicuous or absent at low concentrations. In addition, no FGF23 gene transcript was detected by RT-PCR analysis in non-PMT tumors displaying these staining patterns. Thus, the perinuclear, dot-like staining pattern is reliable phenotypic characteristic of FGF23 expression. Similar perinuclear staining patterns of other markers have been observed during the immunohistochemical analyses of several other tumor types, including Merkel cell carcinoma (cytokeratin 20) [21], desmoplastic small round cell tumors (desmin) [22], and Hodgkin's lymphoma (CD30) [23]. In these tumors, the unique staining patterns are considered to result from clumping of intermediate filaments [21, 22], or the accumulation of protein precursors in the Golgi area. [23]. In PMTs, the punctate staining pattern may result from an accumulation of highly expressed FGF23 precursor molecules in the Golgi apparatus, although further investigations are needed to address the mechanisms underlying the unique intracellular localization pattern of FGF23.

Immunohistochemical examination of FGF23 expression in tumors using a commercially available antibody has been limited thus far [16]. Folpe et al. [3] reported that not only PMTs with TIO, but also PMTs without TIO and some other tumors were immunohistochemically positive for FGF23, using a polyclonal antibody. They scored diffuse cytoplasmic staining as positive and concluded that this staining was not specific. Houang et al. [16] reported that 14 PMTs (all with TIO) and 2 of 40 non-PMT tumors in their series were positive for FGF23 using a different commercially available antibody (polyclonal, 1:100 dilution; Immutopics Inc., San Clemente, CA, USA). The cytoplasmic dot-like expression pattern and the distribution of positive tumor cells observed in their study appeared similar to our results. Although single cases of aneurysmal bone cyst and

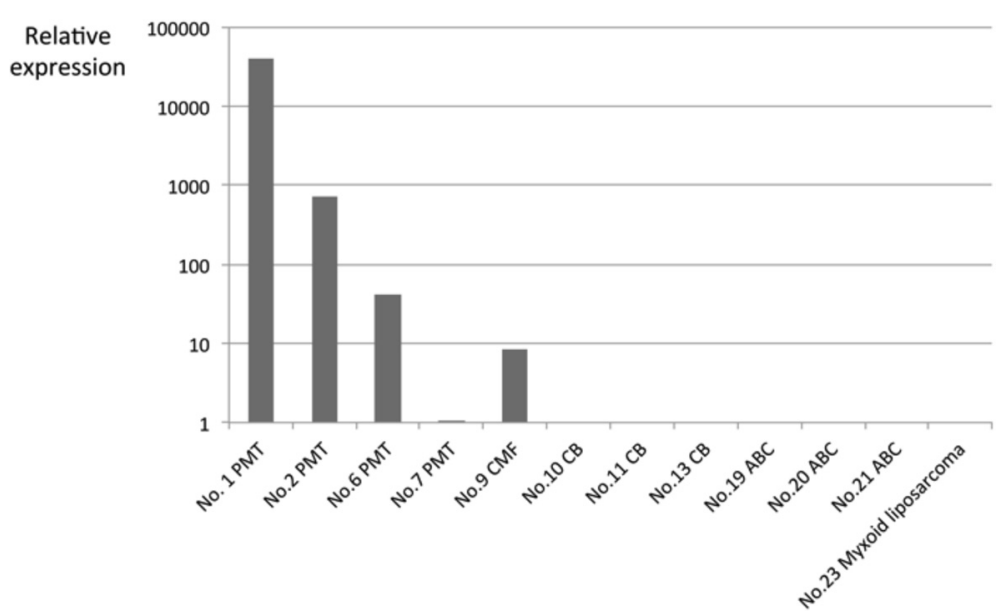

Fig. 6 The relative expression levels of FGF23 in PMTs, as determined by real-time RT-PCR analysis. The expression level of FGF23 was higher in PMTs with $\mathrm{TIO}$ (case Nos. 1 and 2) than in PMTs without TIO (case Nos. 6 and 7) or chondromyxoid fibroma (case No. 9). The FGF23 gene transcript was not identified in the other tumors examined, including myxoid liposarcoma (case No. 23). Expression levels were normalized to GAPDH as an internal control 
osteosarcoma were also positive for FGF23 in their study, it is difficult to conclude that these tumors are unequivocally different from PMTs without TIO, due to their potential morphological overlap.

By RT-PCR analysis, FGF23 gene transcripts were identified not only in the 4 PMTs examined, but also in 1 chondromyxoid fibroma and 1 myxoid liposarcoma. However, no FGF23 gene transcripts were detected in the myxoid liposarcoma sample by real-time RT-PCR. The difference of the primer sequences used in either analysis might contribute to these incongruous results. Because only 1 of the 3 targeted transcripts was detected in the myxoid liposarcoma sample by RT-PCR, a potentially non-functioning splicing variant of FGF23 might have been amplified. Semi-quantitative analysis of the FGF23 gene transcript by real-time RT-PCR showed that the relative expression level tended to be higher in PMTs with TIO than in PMTs without TIO. Tumor cells in the nonphosphaturic variant of PMT may produce FGF23 at a low level. The immunohistochemical expression of FGF23, as well as the clinical manifestation of TIO, may be dependent on the level of secreted FGF23 by tumor cells. The expression level of the FGF23 gene transcript in the chondromyxoid fibroma was also lower than observed in PMTs with TIO; however, it was comparable to PMTs without TIO. These results raise a concern as to whether chondromyxoid fibroma can be completely distinguished from the non-phosphaturic variant of PMT, because it is occasionally challenging to differentiate chondromyxoid fibroma from PMT [24, 25]. Chondromyxoid fibromas typically show a distinct lobular architecture and rarely contain dilated blood vessels. However, the clinical information of TIO may be overlooked, and the histopathological distinction of these tumors may be somewhat arbitrary. Recently, the FN1-FGFR1 fusion gene was identified in PMTs using next-generation RNA sequencing [26], and upregulation of metabotropic glutamate receptor 1 (GRM1) expression through gene fusion and promoter swapping was detected in chondromyxoid fibroma [27]. These distinct molecular profiles may be helpful to differentiate both entities or to diagnose the non-phosphaturic variant of PMT.

\section{Conclusions}

The immunohistochemical expression of FGF23 in PMTs may depend on the level of secreted FGF23 from tumor cells. Thus, immunohistochemical staining for FGF23 may serve as a diagnostic adjunct of PMTs, particularly those with TIO, although further investigations using a greater number of clinical specimens are necessary.

\section{Abbreviations}

FGF23: fibroblast growth factor 23; PMT: phosphaturic mesenchymal tumor; RT-PCR: reverse transcription-polymerase chain reaction; TIO: tumor-induced osteomalacia.

\section{Competing interests}

The authors declare that they have no competing interests.

\section{Authors' contributions}

ES collected case materials, performed the immunohistochemical and molecular-genetics studies, participated in the histological diagnosis and data analysis, and drafted the manuscript. AM conceived of the study and participated in its design and coordination, histological diagnosis, and data analysis, and helped draft the manuscript. RS, KY, HH, and MN collected case materials and participated in the histological diagnoses. TK participated in the histological diagnoses and data analysis. MH coordinated the study, collected case materials, participated in the histological diagnoses and data analysis, and helped draft the final manuscript. All authors read and approved the final manuscript.

\section{Acknowledgements}

We are grateful to Dr. Y. Ishida (Teikyo University Medical Center), Dr. K. Arihiro (Hiroshima University) and Dr. K. Uchihashi (Saga University), who kindly provided materials and clinical information regarding the PMTs.

\section{Author details}

${ }^{1}$ Department of Pathology and Oncology, School of Medicine, University of Occupational and Environmental Health, 1-1 Iseigaoka, Yahatanishi-ku, Kitakyushu 807-8555, Japan. ${ }^{2}$ Kagoshima Occupational and Environmental Health Center, 4-96 Tokaicho, Kagoshima 891-0115, Japan. ${ }^{3}$ National Hospital Organization Kinki-Chuo Chest Medical Center, 1180 Nagasonecho, Kita-ku, Sakai, Osaka 591-8025, Japan.

Received: 28 October 2015 Accepted: 3 March 2016

Published online: 09 March 2016

\section{References}

1. Evans DJ, Azzopardi JG. Distinctive tumours of bone and soft tissue causing acquired vitamin-D-resistant osteomalacia. Lancet. 1972;1:353-4.

2. Weidner N, Santa Cruz D. Phosphaturic mesenchymal tumors. A polymorphous group causing osteomalacia or rickets. Cancer. 1987:59:1442-54.

3. Folpe AL, Fanburg-Smith JC, Billings SD, Bisceglia M, Bertoni F, Cho JY, et al. Most osteomalacia-associated mesenchymal tumors are a single histopathologic entity: an analysis of 32 cases and a comprehensive review of the literature. Am J Surg Pathol. 2004;28:1-30.

4. Fletcher CDM, Bridge JA, Hogendoorn PCW, Mertens F. WHO classification of tumours of soft tissue and bone. 4th ed. Lyon: IARC; 2013. p. 211-2.

5. Goldblum JR, Folpe AL, Weiss SW. Enzinger and Weiss's soft tissue tumors. 6th ed. Philadelphia: Mosby Elsevier; 2014. p. 1018-23.

6. Shimada T, Mizutani S, Muto T, Yoneya T, Hino R, Takeda S, et al. Cloning and characterization of FGF23 as a causative factor of tumor-induced osteomalacia. Proc Natl Acad Sci U S A. 2001;98:6500-5.

7. Bowe $A E$, Finnegan $R$, Jan de Beur SM, Cho J, Levine MA, Kumar R, et al. FGF-23 inhibits renal tubular phosphate transport and is a PHEX substrate. Biochem Biophys Res Commun. 2001;284:977-81.

8. Kolek Ol, Hines ER, Jones MD, LeSueur LK, Lipko MA, Kiela PR, et al. 1a,25Dihydroxyvitamin D3 upregulates FGF23 gene expression in bone: the final link in a renal-gastrointestinal-skeletal axis that controls phosphate transport. Am J Physiol Gastrointest Liver Physiol. 2005;289:G1036-42.

9. Kumar R. New insights into phosphate homeostasis: fibroblast growth factor 23 and frizzled-related protein-4 are phosphaturic factors derived from tumors associated with osteomalacia. Curr Opin Nephrol Hypertens. 2002;11:547-53.

10. Berndt $T$, Craig TA, Bowe AE, Vassiliadis J, Reczek D, Finnegan $R$, et al. Secreted frizzled-related protein 4 is a potent tumor-derived phosphaturic agent. J Clin Invest. 2003;112:785-94.

11. Imanishi Y, Hashimoto J, Ando W, Kobayashi K, Ueda T, Nagata Y, et al. Matrix extracellular phosphoglycoprotein is expressed in causative tumors of oncogenic osteomalacia. J Bone Miner Metab. 2012;30:93-9.

12. Bahrami A, Weiss SW, Montgomery E, Horvai AE, Jin L, Inwards CY, et al. RT-PCR analysis for FGF23 using paraffin sections in the diagnosis of phosphaturic mesenchymal tumors with and without known tumor induced osteomalacia. Am J Surg Pathol. 2009:33:1348-54.

13. Graham R, Krishnamurthy S, Oliveira A, Inwards C, Folpe AL. Frequent expression of fibroblast growth factor-23 (FGF23) mRNA in aneurysmal bone cysts and chondromyxoid fibromas. J Clin Pathol. 2012;65:907-9. 
14. Carter JM, Caron BL, Dogan A, Folpe AL. A novel chromogenic in situ hybridization assay for FGF23 mRNA in phosphaturic mesenchymal tumors. Am J Surg Pathol. 2015;39:75-83.

15. Nelson AE, Bligh RC, Mirams M, Gill A, Au A, Clarkson A, et al. Clinical case seminar: Fibroblast growth factor 23: a new clinical marker for oncogenic osteomalacia. J Clin Endocrinol Metab. 2003;88:4088-94.

16. Houang M, Clarkson A, Sioson L, Elston MS, Clifton-Bligh RJ, Dray M, et al. Phosphaturic mesenchymal tumors show positive staining for somatostatin receptor 2A (SSTR2A). Hum Pathol. 2013;44:2711-8.

17. Ogose A, Hotta T, Emura I, Hatano H, Inoue $Y$, Umezu H, et al. Recurrent malignant variant of phosphaturic mesenchymal tumor with oncogenic osteomalacia. Skeletal Radiol. 2001;30:99-103.

18. Sidell D, Lai C, Bhuta S, Barnes L, Chhetri DK. Malignant phosphaturic mesenchymal tumor of the larynx. Laryngoscope. 2011;121:1860-3.

19. Uchihashi K, Nishijima-Matsunobu A, Matsuyama A, Yamasaki F, Tanabe T, Uemura T, et al. Phosphaturic mesenchymal tumor, nonphosphaturic variant, causing fatal pulmonary metastasis. Hum Pathol. 2013;44:2614-8.

20. Morimoto T, Takenaka S, Hashimoto N, Araki N, Myoui A, Yoshikawa H. Malignant phosphaturic mesenchymal tumor of the pelvis: A report of two cases. Oncol Lett. 2014;8:67-71.

21. Wang TS, Byrne PJ, Jacobs LK, Taube JM. Merkel cell carcinoma: update and review. Semin Cutan Med Surg. 2011:30:48-56.

22. Zhang PJ, Goldblum JR, Pawel BR, Fisher C, Pasha TL, Barr FG Immunophenotype of desmoplastic small round cell tumors as detected in cases with EWS-WT1 gene fusion product. Mod Pathol. 2003;16:229-35.

23. Pileri SA, Ascani S, Lenoncini L, Sabattini E, Zinzani PL, Piccaluga PP, et al. Hodgkin's lymphoma: the pathologist's viewpoint. J Clin Pathol. 2002;55:162-76.

24. Suryawanshi P, Agarwal M, Dhake R, Desai S, Rekhi B, Reddy KB, et al. Phosphaturic mesenchymal tumor with chondromyxoid fibroma-like feature: an unusual morphological appearance. Skeletal Radiol. 2011;40:1481-5.

25. Baker AC, Rezeanu L, O'Laughlin S, Unni K, Klein MJ, Siegal GP. Juxtacortical chondromyxoid fibroma of bone: a unique variant: a case study of 20 patients. Am J Surg Pathol. 2007;31:1662-8.

26. Lee JC, Jeng YM, Su SY, Wu CT, Tsai KS, Lee CH, et al. Identification of a novel FN1-FGFR1 genetic fusion as a frequent event in phosphaturic mesenchymal tumour. J Pathol. 2015;235:539-45.

27. Nord KH, Lilljebjörn H, Vezzi F, Nilsson J, Magnusson L, Tayebwa J, et al. GRM1 is upregulated through gene fusion and promoter swapping in chondromyxoid fibroma. Nat Genet. 2014;46:474-7.

\section{Submit your next manuscript to BioMed Central and we will help you at every step:}

- We accept pre-submission inquiries

- Our selector tool helps you to find the most relevant journal

- We provide round the clock customer support

- Convenient online submission

- Thorough peer review

- Inclusion in PubMed and all major indexing services

- Maximum visibility for your research

Submit your manuscript at www.biomedcentral.com/submit

) Biomed Central 